\title{
Measurement of Trapped Ion Pockets and Control of Ion Instabilities in the Fermilab Antiproton Accumulator
}

\author{
Steven J. Werkema, Kenneth D. Fullett, and Ping Zhou \\ Fermi National Accelerator Laboratory ${ }^{\dagger}$ \\ P.O. Box 500, Batavia, IL 60510-0500
}

\section{Abstract}

Resonant interaction of positively charged, trapped residual gas ions with a negatively charged, intense antiproton beam has been identified as the primary cause of transverse instability in the Fermilab antiproton accumulator. An upgraded ion clearing system was recently installed. This upgrade yielded a significant improvement in machine performance as well as an enhanced capability for studying trapped ion related phenomena. The operational impact and preliminary results from some initial measurements made with this system are presented herein.

\section{INTRODUCTION}

Positively charged ions are produced by the coulomb interaction of the highly relativistic $\bar{p}$ beam particles and residual gas molecules in the accumulator vacuum chamber. The ions created by this process acquire only quasi-thermal energies $(\sim 0.1 \mathrm{eV})$ and are therefore immediately trapped in the space charge potential of the beam. If no counteractive measures are taken, the trapped ions will accumulate in the beam potential well until the beam is completely neutralized. Even with an extensive clearing electrode system, uncleared sites at local beam potential minima, will result in local neutralization of the beam and potentially hazardous pockets of trapped ions.

Pockets of trapped ions have two detrimental effects. First, the neutralization of the beam can cause significant tune shifts. Figure 1 shows the shift in the vertical tune when the clearing voltage in one of the six accumulator sectors is turned off.

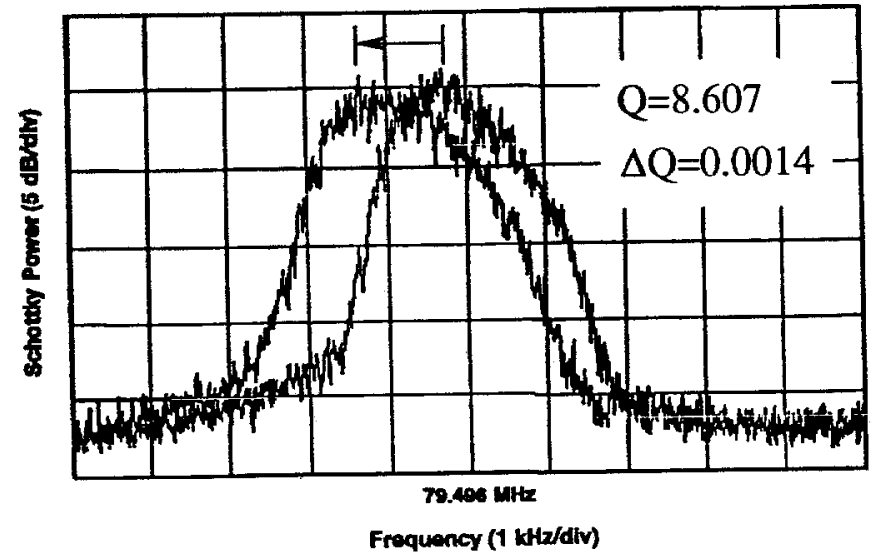

Figure 1. Vertical schottky profile before and after turning off the clearing voltage in accumulator sector 3 .

$\dagger$ Operated by the Universities Research Association, Inc under contract with the U.S. Department of Energy.
The second undesirable consequence of uncleared pockets of trapped ions is that the ions in these pockets can interact resonantly with the beam resulting in large amplitude coherent oscillations and sudden emittance growth [1]. Figure 2 shows the growth in the first 3 coherent dipole modes as the clearing voltage is decreased from $900 \mathrm{~V}$ to $10 \mathrm{~V}$ in one sector of the accumulator. Figure 3 shows a typical sequence of ion induced emittance blowups. The operational exigency to clear trapped ions is obvious.

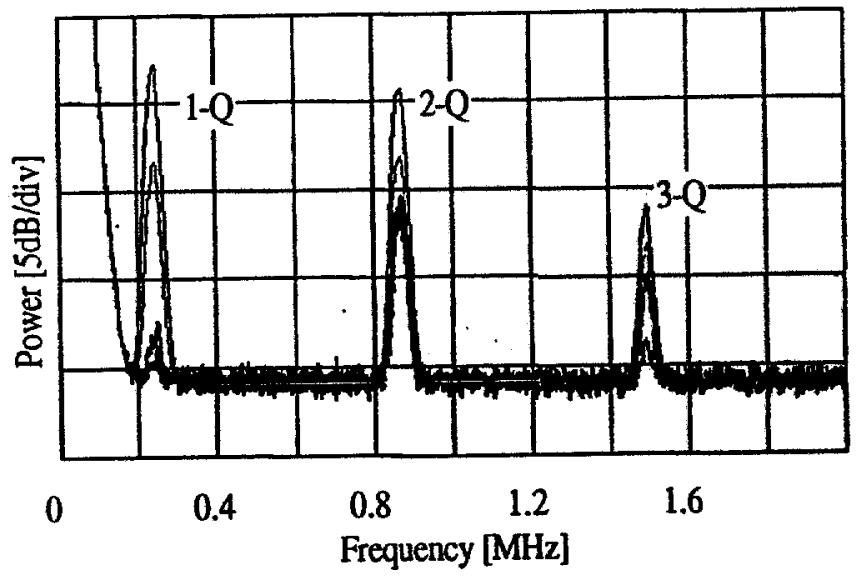

Figure 2. Vertical dipole spectrum showing coherent oscillation at the 1-q, 2-q, and 3-q betatron resonances. As the clearing voltage in accumulator sector 2 is reduced in the sequence $-900 \mathrm{~V}, 200 \mathrm{~V}, 100 \mathrm{~V}, 50 \mathrm{~V}$, and $10 \mathrm{~V}$, the power in each of these lines is seen to grow.

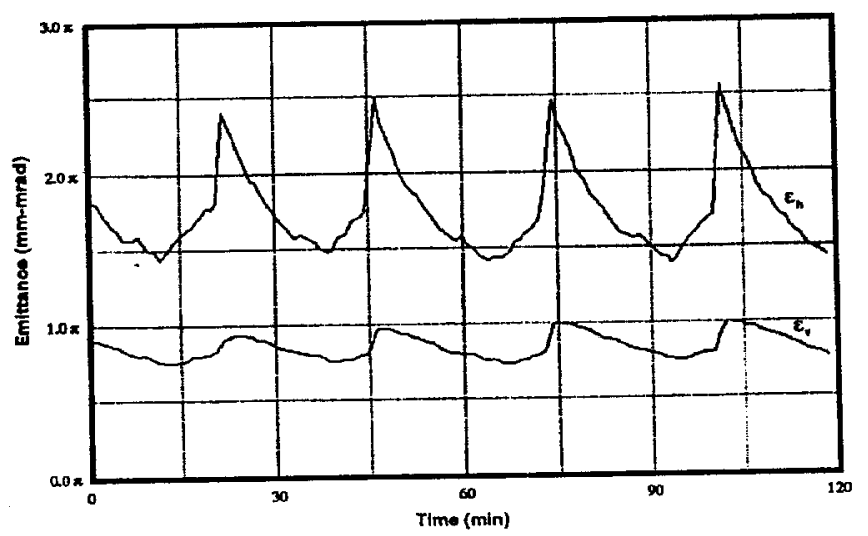

Figure 3. Typical time evolution of trapped ion induced emittance growth.

\section{ION CLEARING ELECTRODE HARDWARE}

The ion clearing system hardware upgrade consists of six power supplies: one for each of the six sectors in the 
Accumulator. The power supplies are individually adjustable through the control system from zero to 1000 volts. Each power supply has ten high voltage output channels. There are an average of two spare channels per sector.

Each channel is connected, by various lengths of coaxial cable, to one or more of the 140 clearing electrodes located throughout the accumulator. Ninety of the electrodes are actually BPM (beam position monitor) pickups; the remaining are dedicated to the ion clearing system. Since the BPM system employs a pre-amplifier at each BPM pickup, it was necessary to insert a one $\mathrm{kV} \mathrm{DC}$ isolation barrier in front of each BPM pre-amplifier and simultaneously maintain its common-mode rejection performance.

Every channel is capable of monitoring its supply current in the range from zero to ten $\mathrm{nA}$. This current is comprised of both ion clearing current and leakage current. The leakage is due to lengths of coaxial cable and a few clearing electrodes of marginal construction.

The supply current resolution is limited by system noise to $6 \mathrm{pA}$. External noise (kicker magnets, pulsed power supplies etc.) can further limit the resolution to $50 \mathrm{pA}$; however, this is dependent upon the operational mode of the accumulator and the location of the clearing electrode and its associated cables.

The clearing electrodes are placed at the most likely locations of trapped ion pockets, which are the minima in the electrostatic potential of the $\bar{p}$ beam. The longitudinal variation of the beam potential well depth depends on the transverse size of the beam and the local dimensions of the vacuum chamber. Figure 4 shows the longitudinal dependence of the beam potential and the deployment of the clearing electrodes for one sector of the accumulator.

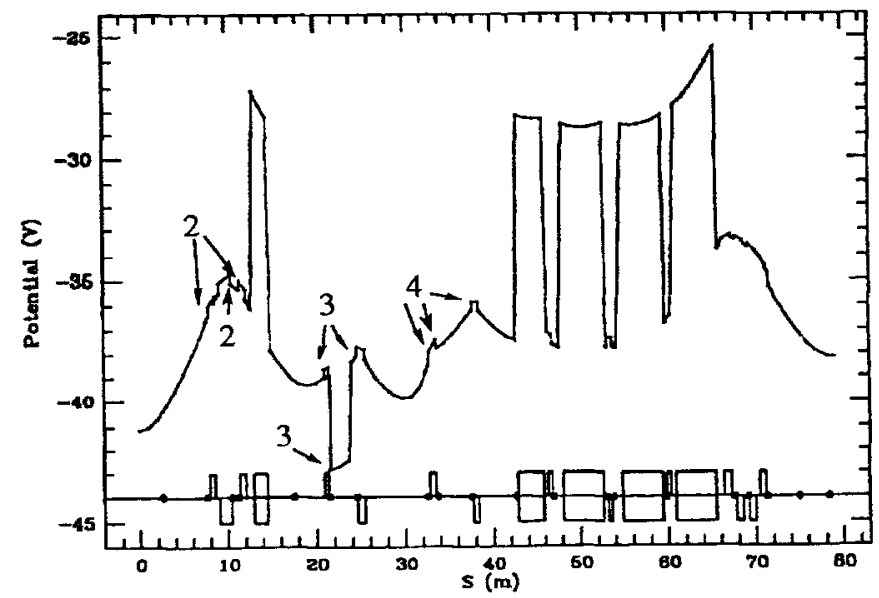

Figure 4. Longitudinal variation of the space charge potential at the center of the beam for accumulator sector 1 . This calculation assumed a $200 \mathrm{~mA} \bar{p}$ beam and transverse emittances of $2 \pi \mathrm{mm} \cdot \mathrm{mrad}$. The black dots indicate the location of clearing electrodes.

\section{OPERATIONAL IMPACT OF THE CLEARING SYSTEM UPGRADE}

The deleterious effects of trapped ions are most apparent during the process of extracting $\bar{p}$ 's for use in the collider.
The number of $\bar{p}$ 's transferred to the collider is critically dependent on the extent to which the beam can be cooled in all three dimensions[2]. Prior to the clearing upgrade, relatively small longitudinal densities ( $\leqslant 7 \times 10^{10} \bar{p}^{\prime} \mathrm{s} / \mathrm{eV} \cdot \mathrm{sec}$ ) would result in transverse instabilities. These instabilities would preclude further transverse cooling of the beam. The advent of the ion clearing upgrade increased the achievable longitudinal density by a factor of nearly 2 for horizontal emittances near the threshold for efficient transfer into the collider (see Figure 5).

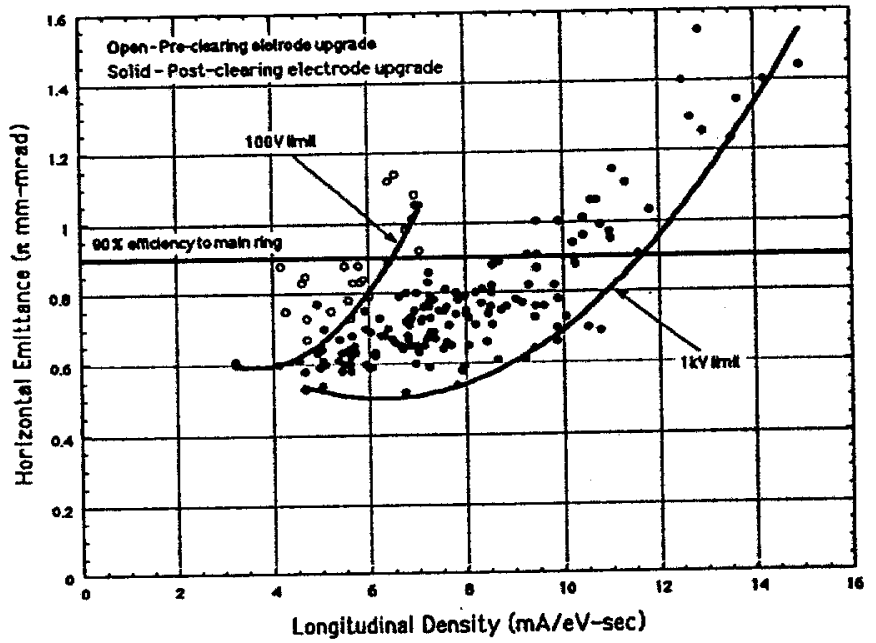

Figure 5. Transverse beam size versus longitudinal density before and after the ion clearing upgrade.

The ion clearing upgrade eliminated the periodic emittance blowup phenomena shown in Figure 3 for $\bar{p}$ stacks of less than $120 \times 10^{10}$.

\section{MEASUREMENTS OF TRAPPED ION BEHAVIOR}

At the time of this writing, the ion current readback has been in operation for 5 weeks. During this time there have been several opportunities to use this system to study trapped ion phenomena. Some preliminary data from these studies are presented here.

\section{A. Longitudinal Mobility of Trapped Ions}

A measurement of the longitudinal mobility of trapped ions is of interest in evaluating the effectiveness of the clearing system and provides an assessment of the validity of the beam space charge potential calculation.

The method used in this study is to turn off the high voltage on one channel of the clearing system. When this is done, the pockets cleared by the clearing electrodes connected to that channel will begin to fill up with ions. As these pockets fill up, the beam becomes partially neutralized at these sites. The local minima in the beam potential quickly disappear and ions produced subsequently will not be locally trapped, but will be free to move in accordance with the longitudinal gradient of the beam space charge potential. The 
ion current readback system is used to determine where the ions go when the local pockets are neutralized in this manner.

Figure 6 shows the clearing current readback system response to turning off the high voltage on channel 3 in accumulator sector 1 . The clearing current in the adjacent channels (channels 2 and 4) increase; with the greatest increase going to channel 4 . Figure 4 shows that there is a large potential barrier between the channel 3 electrodes and the channel 2 electrodes (due to a change in beam pipe diameter). It is, therefore, expected that the majority of ions produced after the pockets at the channel 3 electrodes fill up will indeed go to the channel 4 electrodes.

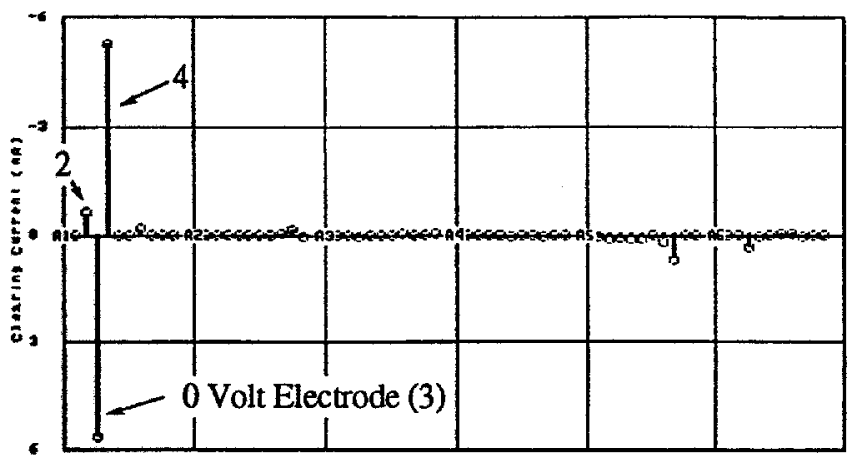

Figure 6. Display of the clearing current readback of all instrumented channels in the accumulator. The current values are differences: Channel 3 OFF - Channel 3 ON. A negative value indicates a net increase in charge cleared.

It is also apparent that the number of ions cleared by the channel 3 electrodes before the voltage was turned off is the same as the number of ions produced at the channel 3 electrode sites but cleared by channels 2 and 4 when the voltage is turned off.

This test was performed on each high voltage channel in the clearing system. The result shown in Figure 6 is typical of what was seen in other channels with a few exceptions. The exceptions include: (1) devices affecting the beam space charge potential which were not incorporated into the potential calculation (e.g. stochastic cooling pickups and kickers), (2) ineffective clearing electrodes, and (3) some genuine mysteries.

The study of trapped ion longitudinal mobility will be continued by attempting to measure the ion longitudinal drift velocities. The bandwidth of the ion current readback system is $10 \mathrm{~Hz}$, which is inadequate for this measurement (the expected velocities are of order several hundred meters/sec). A portable readback system with a bandwidth of $2 \mathrm{kHz}$ is presently being built to facilitate this measurement.

\section{B. Stabilization by Weak RF Bunching}

Bunching a small fraction of the accumulator $\bar{p}$ beam with RF has been observed to suppress transverse instability[2]. A study has been initiated to ascertain the mechanism by which this stabilization occurs. One possibility is that pockets of trapped ions, which are inaccessible to the clearing electrode system, are being cleared by the longitudinal modulation of the beam intensity caused by the RF. The clearing current readback system was used to locate these pockets. Figure 7 shows the clearing current readback response to bunching the beam in a $1.5 \mathrm{eV} \cdot \mathrm{sec}$ bucket with an $\mathrm{h}=2$ suppressed bucket RF system. Approximately $15 \%$ of the beam is contained in the RF bucket.

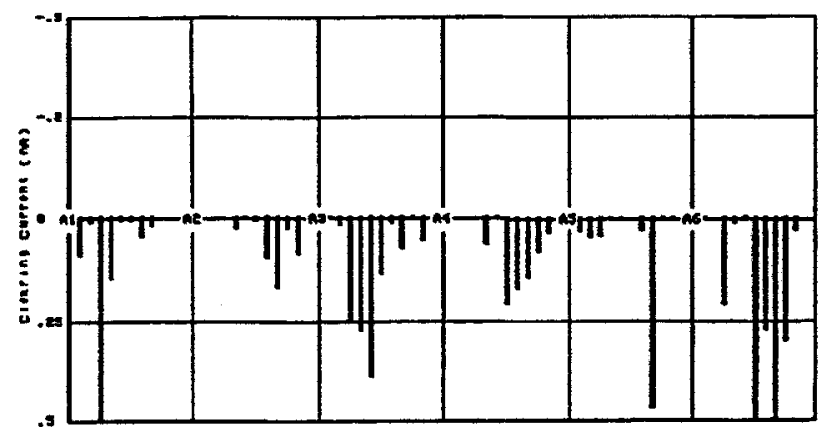

Figure 7. Display of the clearing current readback of all instrumented channels in the accumulator. The current values are differences: RF OFF - RF ON. A positive value indicates a reduction in clearing current.

The clearing current readback indicates a reduction in ion current at roughly the same locations in each sector of the accumulator. A reduction in clearing current is an indication that ions from a nearby site, which were previously drifting to a clearing electrode, are being removed by the effects of RF bunching to the vacuum chamber walls. Generally, it appears that the sites being cleared by RF bunching are in the long straight sections of the accumulator.

Bunching the beam with RF is beneficial even with very small buckets. Applying 5 Volts is nearly as effective as 20 Volts. Moreover, the beam remains stable for a long time (hours) after the RF is turned off.

This effect is far from understood. Much work, both analytical and experimental, remains to be done before any further explanation can be offered.

\section{REFERENCES}

[1] P. Zhou, P. Colestock, and S.J. Werkema, Trapped Ions and Beam Coherent Instability. These proceedings (1993).

[2] S.J. Werkema, D.W. Peterson, and P. Zhou, Transverse Emittance Growth in the Fermilab Antiproton Accumulator with High-Current Antiproton Stacks. These proceedings (1993). 